\title{
A simple new test for slope homogeneity in panel data models with interactive effects ${ }^{1}$
}

\author{
September 11, 2015 \\ Tomohiro Ando \\ Jushan Bai \\ University of Melbourne \\ Columbia University
}

\begin{abstract}
We consider the problem of testing for slope homogeneity in high-dimensional panel data models with a factor error structure. We consider the Swamy-type test for slope homogeneity by incorporating interactive fixed effects. We show that the proposed test statistic is asymptotically normal. Our test allows the explanatory variables to be correlated with the unobserved factors, factor loadings, or with both. Monte Carlo simulations demonstrate that the proposed test has good size control and good power.
\end{abstract}

Key words: Cross-sectional dependence, Endogenous regressors, Slope homogeneity

JEL Classification Codes: C12, C23.

\footnotetext{
${ }^{1}$ Tomohiro Ando: Melbourne Business School, University of Melbourne, 200 Leicester Street, Carlton, Victoria 3053, Australia E-mail: T.Ando@mbs.edu. Ando's research is partially supported by the Trust Companies Association of Japan.

Jushan Bai: Department of Economics, Columbia University, 420 West 118 Street, 1022 International Affairs Building, New York, NY, 10027. E-mail: jb3064@columbia.edu; School of Finance, Nankai University, China. Bai's research is partially supported by the NSF grant SES1357598.
} 


\section{Introduction}

This paper considers the testing of slope homogeneity for high-dimensional panel data models. Testing slope homogeneity is useful for empirical studies. In finance, for example, testing asset pricing models, including the capital asset pricing model $^{2}$ and the multiple factor pricing models, ${ }^{3}$ is related to testing homogeneity. ${ }^{4}$ There are a number of studies on testing for slope homogeneity in panel data models, including Pesaran et al. (1996), Phillips and Sul (2003), Pesaran and Yamagata (2008), Blomquist and Westerlund (2013), and Su and Chen (2013).

Pesaran and Yamagata's (2008) testing procedure is useful in the sense that it treats a high-dimensional panel data model where the number of cross-sectional units $N$ and the time series dimension $T$ are large. However, the test does not allow crosssectional and serial correlations in the errors. To deal with the serially correlated errors, Blomquist and Westerlund (2013) extended their test to the case when the errors are heteroskedastic and/or serially correlated in an unknown fashion. However, the test still does not deal with the practically relevant case of cross-sectional dependence. Moreover, these tests do not allow dependence between the regressors and the unobservable errors.

To deal with these problems, we propose a new simple test that accommodates cross-sectional dependence by using the results of Bai (2009), Song (2013), and Ando and Bai (2015). These studies considered panel data models with interactive fixed effects. Our proposed test statistic, denoted by $\hat{\Gamma}$, is a modified version of Swamy's (1970) test statistic. ${ }^{5}$ An advantage of our testing procedure is that it provides a robust test under cross-sectionally correlated errors with heteroskedasticity. Furthermore, the proposed test works even when the set of regressors and the unobservable errors that contain the factor structure are correlated. We investigate the asymptotic distribution of our test statistic, and show that the test has a standard normal distribution as $N, T \rightarrow \infty$ such that $\sqrt{T} / N \rightarrow 0$. Monte Carlo experiments show that the proposed test tends to have the correct size and satisfactory power as $N, T \rightarrow \infty$.

Recently, Su and Chen (2013) proposed a residual-based LM test for slope homogeneity in high-dimensional panel data models with interactive fixed effects. In general, $\mathrm{Su}$ and Chen's test works well. But there is a tendency of size distortion when $N$ is much larger than $T$. One possible explanation is that their assumptions $N^{3 / 4} / T \rightarrow 0$ and $T^{2 / 3} / N \rightarrow 0$ imply a relatively narrow band between $N$ and $T$. Usually, these assumptions are sufficient conditions, and they are not necessarily required in practice. But Monte Carlo experiments do reveal that these assumptions between $N$ and $T$ ap-

\footnotetext{
${ }^{2}$ See, e.g., Sharpe (1965) and Lintner (1965).

${ }^{3}$ See, e.g., Fama and French (1992) and Carhart (1997).

${ }^{4}$ In this case, it involves testing intercept homogeneity, which is a special case of the test considered in this paper.

${ }^{5}$ Pesaran and Yamagata's (2008) test is also based on Swamy's testing principle.
} 
pear to be crucial. In contrast, the proposed test in this paper provides the correct size control even when $N$ is much larger than $T$. Our test also exhibits very good powers.

The proposed test here requires $\sqrt{N} / T \rightarrow 0$, which is weaker than the condition imposed by $\mathrm{Su}$ and Chen (2013). The latter requires $N^{3 / 4} / T \rightarrow 0$ and $T^{2 / 3} / N \rightarrow 0$. Thus, it is possible to face a situation where the relationship between $N$ and $T$ satisfies our assumptions, while the conditions of Su and Chen (2013) are not met. Through a Monte Carlo simulation, it is shown that, under such situations, the proposed test still provides the correct size, while a size distortion is observed by Su and Chen's (2013) test.

Notation. Let $\|A\|=\left[\operatorname{tr}\left(A^{\prime} A\right)\right]^{1 / 2}$ be the usual norm of the matrix $A$, where "tr" denotes the trace of a square matrix. The equation $a_{n}=O\left(b_{n}\right)$ states that the deterministic sequence $a_{n}$ is at most of order $b_{n}, c_{n}=O_{p}\left(d_{n}\right)$ states that the random variable $c_{n}$ is at most of order $d_{n}$ in terms of probability, and $c_{n}=o_{p}\left(d_{n}\right)$ is of a smaller order in terms of probability. All asymptotic results are obtained under a large number of units $N$ and a large number of time periods $T$.

The outline of the rest of the paper is as follows. Section 2 proposes the $\hat{\Gamma}$ test statistic, a modified version of Swamy's (1970) test statistic, and derives its asymptotic distribution. In Section 3, we conduct Monte Carlo experiments to evaluate the finite sample performance of the proposed test. Some concluding remarks are provided in Section 4.

\section{A new procedure for testing slope homogeneity}

Consider the following high-dimensional panel data model, with a large number of cross-sectional units $N$ and a large number of time periods $T$

$$
\boldsymbol{y}_{i}=X_{i} \boldsymbol{\beta}_{i}+\boldsymbol{u}_{i}, \quad i=1, \ldots, N,
$$

where $\boldsymbol{y}_{i}=\left(y_{i 1}, \ldots, y_{i T}\right)^{\prime} X_{i}=\left(\boldsymbol{x}_{i 1}, \ldots, \boldsymbol{x}_{i T}\right)^{\prime}, \boldsymbol{u}_{i}=\left(u_{i 1}, \ldots, u_{i T}\right)^{\prime}$. Here, each $\boldsymbol{x}_{i t}$ is a $p \times 1$ vector of observable regressors, $\boldsymbol{\beta}_{i}$ is a $p \times 1$ vector of unknown slope coefficients, and $u_{i t}$ is an idiosyncratic error. The null hypothesis of interest in this paper is

$$
H_{0}: \boldsymbol{\beta}_{1}^{0}=\boldsymbol{\beta}_{2}^{0}=\cdots=\boldsymbol{\beta}_{N}^{0}=\boldsymbol{\beta}^{0} \text { for some } \boldsymbol{\beta}^{0} .
$$

The alternative hypothesis is

$$
H_{1}: \boldsymbol{\beta}_{i}^{0} \neq \boldsymbol{\beta}_{j}^{0} \text { for a nonzero fraction of pairwise slopes for } i \neq j \text {. }
$$

There are several procedures that can be used to test the null hypothesis. Although one may consider the standard $F$ statistic, this test is valid for a fixed $N$, while this paper focuses on high-dimensional panel data models with large $N$ and $T$. In this section, we provide a literature review of the test of slope homogeneity for high-dimensional panel data models. 


\section{$2.1 \quad$ Model}

Consider a high-dimensional panel data model, with a large number of cross-sectional units $N$, and a large number of time periods $T, \boldsymbol{y}_{i}=X_{i} \boldsymbol{\beta}_{i}+\boldsymbol{u}_{i}$ in (1). In this paper, we assume that the error term contains multifactor structures:

$$
\boldsymbol{u}_{i}=F \boldsymbol{\lambda}_{i}+\varepsilon_{i}, \quad, i=1, \ldots, N
$$

where

$$
F=\left(\begin{array}{c}
\boldsymbol{f}_{1}^{\prime} \\
\boldsymbol{f}_{2}^{\prime} \\
\vdots \\
\boldsymbol{f}_{T}^{\prime}
\end{array}\right), \boldsymbol{\lambda}_{i}=\left(\begin{array}{c}
\lambda_{i 1} \\
\lambda_{i 2} \\
\vdots \\
\lambda_{i r}
\end{array}\right), \boldsymbol{\varepsilon}_{i}=\left(\begin{array}{c}
\varepsilon_{i 1} \\
\varepsilon_{i 2} \\
\vdots \\
\varepsilon_{i T}
\end{array}\right)
$$

where $\boldsymbol{f}_{t}$ is an $r \times 1$ vector of unobservable common factors, $\boldsymbol{\lambda}_{i}$ is the factor loading, and $\varepsilon_{i t}$ are the idiosyncratic errors. In the next section, we describe the assumptions under the null and alternative hypotheses.

\subsection{Assumptions}

We state the assumptions needed for the asymptotic analysis.

\section{Assumption A: Common factors}

The common factors satisfy $E\left\|\boldsymbol{f}_{t}\right\|^{4}<\infty$. Furthermore, $T^{-1} \sum_{t=1}^{T} \boldsymbol{f}_{t} \boldsymbol{f}_{t}{ }^{\prime} \rightarrow \Sigma_{F}$ as $T \rightarrow \infty$, where $\Sigma_{F}$ is an $r \times r$ positive definite matrix.

\section{Assumption B: Factor loadings}

The factor-loading matrix $\Lambda=\left[\boldsymbol{\lambda}_{1}, \ldots, \boldsymbol{\lambda}_{N}\right]^{\prime}$ satisfies $E\left\|\boldsymbol{\lambda}_{i}^{4}\right\|<\infty$ and $\| N^{-1} \Lambda^{\prime} \Lambda-$ $\Sigma_{\Lambda} \| \rightarrow \mathbf{0}$ as $N \rightarrow \infty$, where $\Sigma_{\Lambda}$ is an $r \times r$ positive definite matrix.

\section{Assumption C: Error terms}

There exists a positive constant $C<\infty$ such that for all $N$ and $T$,

(1): $E\left[\varepsilon_{i t}\right]=0, E\left[\left|\varepsilon_{i t}\right|^{8}\right]<C$ for all $i$ and $t$;

(2): $\varepsilon_{i t}$ and $\varepsilon_{j s}$ are independent, for $i \neq j$ and $t \neq s$.

(3): For every $(s, t), E\left[\left|N^{-1 / 2} \sum_{i=1}^{N}\left(\varepsilon_{i s} \varepsilon_{i t}-E\left[\varepsilon_{i s} \varepsilon_{i t}\right]\right)\right|^{4}\right]<C$.

(4): $\varepsilon_{i t}$ is independent of $\boldsymbol{x}_{j s}, \boldsymbol{\lambda}_{i}$, and $\boldsymbol{f}_{s}$ for all $i, j, t, s$.

\section{Assumption D: Explanatory variables}

We assume $E\left\|\boldsymbol{x}_{i t}\right\|^{4}<C$. The $p \times p$ matrix $\frac{1}{T}\left[X_{i}^{\prime} M_{F^{0}} X_{i}\right]$ is positive definite, where $M_{F}=I-F\left(F^{\prime} F\right)^{-1} F^{\prime}$, and $M_{F_{0}}$ is equal to $M_{F}$ evaluated at the true common factors 
$F^{0}$. Furthermore, we define $A_{i}=\frac{1}{T} X_{i}^{\prime} M_{F} X_{i}, B_{i}=\left(\boldsymbol{\lambda}_{i} \boldsymbol{\lambda}_{i}^{\prime}\right) \otimes I_{T}, C_{i}=\frac{1}{\sqrt{T}} \boldsymbol{\lambda}_{i}^{\prime} \otimes\left(X_{i}^{\prime} M_{F}\right)$. Let $\mathcal{A}$ be the collection of $F$ such that $\mathcal{A}=\left\{F: F^{\prime} F / T=I\right\}$. We assume

$$
\inf _{F \in \mathcal{A}}\left[\frac{1}{N} \sum_{i=1}^{N} E_{i}(F)\right] \quad \text { is positive definite, }
$$

where $E_{i}(F)=B_{i}-C_{i}^{\prime} A_{i}^{-} C_{i}$ and $A_{i}^{-}$is the generalized inverse of $A_{i}$.

Assumption E: Central limit theory. As $T$ goes to infinity,

$$
\Omega_{i}^{-1 / 2} \frac{1}{\sqrt{T}} X_{i}^{\prime} M_{F^{0}} \varepsilon_{i} \rightarrow_{d} N\left(\mathbf{0}, I_{p}\right)
$$

where $\Omega_{i}=E\left[X_{i}^{\prime} M_{F^{0}} \varepsilon_{i} \varepsilon_{i}^{\prime} M_{F^{0}} X_{i}\right] / T$.

\section{Assumption F: Joint Limit}

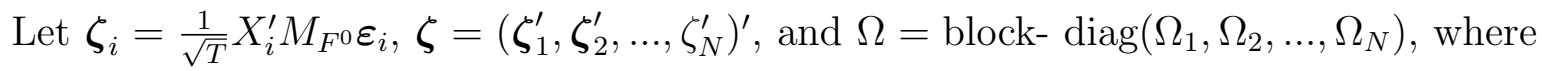
$\Omega_{i}$ is defined in Assumption E. We assume, as $N, T \rightarrow \infty$,

$$
\frac{\boldsymbol{\zeta}^{\prime} \Omega^{-1} \boldsymbol{\zeta}-N p}{\sqrt{2 N p}} \rightarrow N(0,1)
$$

Remark 1 Assumptions A and B above are commonly imposed on the panel data model (1). The full rank assumptions of $\Sigma_{F}$ and $\Sigma_{\Lambda}$ imply the number of common factors is $r$. Assumption $\mathrm{C}$ allows heteroskedasticity in the idiosyncratic errors $\varepsilon_{i}$. Assumptions D and E above are imposed for consistency and asymptotic distributions of the slope coefficients (see Bai (2009), Song (2013), Ando and Bai (2015)).

Remark 2 The variables in Assumption E do not involve any estimated quantities, they are random variables from the true model. Because $\varepsilon_{i}$ is assumed to be independent of $X_{i}$ and $F^{0}$, it follows that the conditional mean of $\frac{1}{\sqrt{T}} X_{i}^{\prime} M_{F^{0}} \varepsilon_{i}$, conditional on $\left(X_{i}, F^{0}\right)$, is equal to 0 , and the conditional and unconditional variance of $\Omega_{i}^{-1 / 2} \frac{1}{\sqrt{T}} X_{i}^{\prime} M_{F^{0}} \varepsilon_{i}$ is an identity matrix. Assumption E imposes the central limit theorem for $\boldsymbol{\zeta}_{i}$. Furthermore, the expected value of $\boldsymbol{\zeta}_{i}^{\prime} \Omega_{i}^{-1} \boldsymbol{\zeta}_{i}$ is equal to $p$, thus the expected value of $\boldsymbol{\zeta}^{\prime} \Omega^{-1} \boldsymbol{\zeta}$ is equal to $N p$. The latter follows from the assumption that $\varepsilon_{i}$ is independent of $\varepsilon_{j}$ for $i \neq j$, thus $\Omega$ is the variance-covariance matrix of $\boldsymbol{\zeta}$. Under a fixed $N$, Assumption E implies Assumption F. But because $N$ is large in this paper, a stronger condition is needed. Assumption $\mathrm{F}$ assumes the joint limit, as $N$ and $T$ go to infinity, to be normal. This is a high level assumption to simplify the argument.

We consider estimating the model (1) with factor structure (2) under the null and alternative hypotheses, respectively. Under the null hypothesis, we can estimate the common slope coefficient $\boldsymbol{\beta}$ by using the procedure in Bai (2009). Under the alternative, 
we employ the estimation procedure in Song (2013) and Ando and Bai (2015). Given the number of common factors $r$, we minimize the least-squares objective function

$$
\ell\left(\boldsymbol{\beta}_{1}, \ldots, \boldsymbol{\beta}_{N}, F, \Lambda\right)=\sum_{i=1}^{N}\left\|\boldsymbol{y}_{i}-X_{i} \boldsymbol{\beta}_{i}-F \boldsymbol{\lambda}_{i}\right\|^{2}
$$

subject to the constraints on the factors and its loadings (see Connor and Korajczyk (1986), Bai and Ng (2002), Bai (2009)). The number of common factors can be selected by the $C_{p}$ criterion, proposed in Ando and Bai (2015). Thus, we can compare the restricted and unrestricted estimators of the slope coefficients.

\subsection{A new slope homogeneity test}

To test slope homogeneity, we consider Swamy's test statistic. Swamy's (1970) test of slope homogeneity calculates the dispersion of individual slope estimates from a suitable pooled estimator (also see Pesaran and Yamagata (2008)). With a factor structure, Swamy's test statistic for slope homogeneity takes the form

$$
\hat{\Gamma}=\frac{T\left(\hat{\boldsymbol{\beta}}-\overline{\boldsymbol{\beta}}_{1_{N}}\right)^{\prime}\left(\hat{S}-\frac{1}{N} \hat{L}^{\prime}\right) \hat{\Omega}^{-1}\left(\hat{S}-\frac{1}{N} \hat{L}\right)\left(\hat{\boldsymbol{\beta}}-\overline{\boldsymbol{\beta}}_{1_{N}}\right)-N p}{\sqrt{2 N p}},
$$

where $\hat{\boldsymbol{\beta}}^{\prime}=\left(\hat{\boldsymbol{\beta}}_{1}^{\prime}, \ldots, \hat{\boldsymbol{\beta}}_{N}^{\prime}\right), \overline{\boldsymbol{\beta}}_{1_{N}}^{\prime}=\left(\overline{\boldsymbol{\beta}}^{\prime}, \ldots, \overline{\boldsymbol{\beta}}^{\prime}\right), \overline{\boldsymbol{\beta}}=\sum_{i=1}^{N} \hat{\boldsymbol{\beta}}_{i} / N$, and $\hat{\boldsymbol{\beta}}_{i}$ are obtained by minimizing (4), $\hat{S}$ is an $N p \times N p$ block diagonal matrix with $i$ th block $\left(X_{i}^{\prime} M_{\hat{F}} X_{i}\right) / T$, and $\hat{L}$ is an $N p \times N p$ matrix with $i j$-th block $\hat{a}_{i j}\left(X_{i}^{\prime} M_{\hat{F}} X_{j}\right) / T$ with $\hat{a}_{i j}=\hat{\boldsymbol{\lambda}}_{j}^{\prime}\left(\hat{\Lambda}^{\prime} \hat{\Lambda} / N\right)^{-1} \hat{\boldsymbol{\lambda}}_{j}$ and $\hat{\Omega}$ is the variance-covariance estimator of $\Omega$, defined in Assumption F. This is, $\Omega$ is block-diagonal matrix consisting of $\Omega_{i}$ as its block-diagonal elements, where $\Omega_{i}$ is the variance-covariance matrix of $\xi_{i}=X_{i}^{\prime} M_{F_{0}} \varepsilon_{i} / \sqrt{T}, i=1, \ldots, N$. The following theorem provides the asymptotic distribution of $\hat{\Gamma}$ under the null hypothesis.

Theorem 1 Suppose that Assumptions $A-F$ and $\sqrt{T} / N \rightarrow 0$ hold. Then, under $H_{0}$,

$$
\hat{\Gamma} \rightarrow N(0,1) \quad \text { in distribution, }
$$

as $T, N \rightarrow \infty$.

The proof of Theorem 1 is provided in the Appendix. The proposed test is simple to implement as it has a limiting $N(0,1)$ distribution.

Remark 3 Under the null $H_{0}$, we need the value of the true common slope coefficients $\boldsymbol{\beta}^{0}$, for which we use $\overline{\boldsymbol{\beta}}=\sum_{i=1}^{N} \hat{\boldsymbol{\beta}}_{i} / N$ where $\hat{\boldsymbol{\beta}}_{i}$ are obtained by minimizing (4). Note that we can also employ Bai's (2009) estimator $\hat{\boldsymbol{\beta}}$. Because these two provide similar results, we thus report only the use of $\overline{\boldsymbol{\beta}}$. 
Remark 4 This remark explains the intuition behind the construction of the test statistics. In the absence of the factor structure, or if the factor process $f_{t}$ is observable, then the term $N^{-1} L$ will not appear in (5), and the resulting statistic would be the standard Swamy-type test. The main insight of this paper is that in the presence of factor structure, the term $N^{-1} L$ plays a central role in correcting the bias caused by estimating the factor structure. Without this term, the traditional Swamy-type test would be severely biased, especially when one of the dimensions is small. The reason is the following. It can be shown that, also see Song (2013),

$$
\sqrt{T}\left(\hat{\boldsymbol{\beta}}_{i}-\boldsymbol{\beta}_{i}^{0}\right)=S_{i i}^{-1} \boldsymbol{\zeta}_{i}+S_{i i}^{-1} \frac{1}{N} \sum_{j=1}^{N} L_{i j} \sqrt{T}\left(\hat{\boldsymbol{\beta}}_{j}-\boldsymbol{\beta}_{j}^{0}\right)+o_{p}(1), \quad i=1, \ldots, N
$$

where $S_{i i}=\left(X_{i}^{\prime} M_{F^{0}} X_{i}\right) / T, L_{i j}=a_{i j}\left(X_{i}^{\prime} M_{F^{0}} X_{j}\right) / T$ with $a_{i j}=\boldsymbol{\lambda}_{j}^{\prime}\left(\Lambda^{\prime} \Lambda / N\right)^{-1} \boldsymbol{\lambda}_{j}$. Note that for each given $i$, the limiting behavior of $\sqrt{T}\left(\hat{\boldsymbol{\beta}}_{i}-\boldsymbol{\beta}_{i}^{0}\right)$ is determined by the dominating term $S_{i i}^{-1} \boldsymbol{\zeta}_{i}$, and the second term on the right hand side of (6) is negligible. This is because, as an average of $\sqrt{T}\left(\hat{\boldsymbol{\beta}}_{j}-\boldsymbol{\beta}_{j}\right)$ over all $j$, the second term is of order $O_{p}\left(N^{-1 / 2}\right)$. But the homogeneity test involves the entire vector $\hat{\boldsymbol{\beta}}=\left(\hat{\boldsymbol{\beta}}_{1}^{\prime}, \ldots, \hat{\boldsymbol{\beta}}_{N}^{\prime}\right)^{\prime}$. Equation (6) implies, stacking over $i$,

$$
\sqrt{T}(\hat{\boldsymbol{\beta}}-\boldsymbol{\beta})=S^{-1} \zeta+S^{-1} \frac{1}{N} L \sqrt{T}(\hat{\boldsymbol{\beta}}-\boldsymbol{\beta})+o_{p}(1)
$$

Both sides of the equation depend on the entire vector $\hat{\boldsymbol{\beta}}$. Combining $\hat{\boldsymbol{\beta}}-\boldsymbol{\beta}$ from both sides produces a more precise asymptotic representation for $\hat{\boldsymbol{\beta}}-\boldsymbol{\beta}$; see (12) in the appendix. The better asymptotic representation gives rise to a better performance of the test statistic.

To compute the test statistic $\hat{\Gamma}$, we need to estimate the variance-covariance matrix of $X_{i}^{\prime} M_{F_{0}} \varepsilon_{i} / \sqrt{T}, \Omega_{i}$. The following provides a practical calculation method for $\Omega_{i}$.

\section{Case 1: Homoskedastic errors over $i$ and $t$}

In this case, $\Omega_{i}$ is given as $\Omega_{i}=\sigma^{2} S_{i i}$ with $\sigma^{2}=\operatorname{Var}\left(\varepsilon_{i t}\right)$. In the absence of serial correlation and heteroskedasticity, the common variance can be estimated by

$$
\hat{\sigma}^{2}=\frac{1}{N T-N p-(N+T) r} \sum_{i=1}^{N} \sum_{t=1}^{T}\left(y_{i t}-\boldsymbol{x}_{i t}^{\prime} \hat{\boldsymbol{\beta}}_{i}-\hat{\boldsymbol{f}}_{t}^{\prime} \hat{\boldsymbol{\lambda}}_{i}\right)^{2}
$$

Case 2: Heteroskedastic errors over $i$

The $i$-th block diagonal element of $\Omega, \Omega_{i}$, is given as $\Omega_{i}=\sigma_{i}^{2} S_{i i}$ with $\sigma_{i}^{2}=\operatorname{Var}\left(\varepsilon_{i t}\right)$. The variance can be estimated as

$$
\hat{\sigma}_{i}^{2}=\frac{1}{T-p} \sum_{t=1}^{T}\left(y_{i t}-\boldsymbol{x}_{i t}^{\prime} \hat{\boldsymbol{\beta}}_{i}-\hat{\boldsymbol{f}}_{t}^{\prime} \hat{\boldsymbol{\lambda}}_{i}\right)^{2}
$$


Case 3: Heteroskedastic errors over $i$ and $t$

If the errors $\varepsilon_{i t}$ are heteroskedastic over $i$ and $t$ (i.e., $E\left[\varepsilon_{i t}\right]=\sigma_{i t}^{2}$ ), then $\hat{\Omega}_{i}=T^{-1} \sum_{t=1}^{T} \hat{\boldsymbol{x}}_{i t} \hat{\boldsymbol{x}}_{i t}^{\prime} \hat{\varepsilon}_{i t}^{2}$, where $\hat{\boldsymbol{x}}_{i t}$ is the $t$-th row of $M_{\hat{F}} X_{i}$. Furthermore, with serial correlation, we can also use the method of Blomquist and Westerlund (2013) to compute $\Omega_{i}$.

Remark 5 A simpler version of the test statistic is

$$
\tilde{\Gamma}=\frac{\sum_{i=1}^{N} T\left(\hat{\boldsymbol{\beta}}_{i}-\overline{\boldsymbol{\beta}}\right)^{\prime} S_{i i} \hat{\Omega}_{i}^{-1} S_{i i}\left(\hat{\boldsymbol{\beta}}_{i}-\overline{\boldsymbol{\beta}}\right)\left[1-\hat{\boldsymbol{\lambda}}_{i}^{\prime}\left(\hat{\Lambda}^{\prime} \hat{\Lambda}\right)^{-1} \hat{\boldsymbol{\lambda}}_{i}\right]^{2}-N p}{\sqrt{2 N p}} .
$$

Under Assumptions $\mathrm{A}-\mathrm{F}$ and $\sqrt{T} / N \rightarrow 0$, the $\tilde{\Gamma}$ asymptotically follows the standard normal distribution under the null $H_{0}$.

\section{Simulation}

In this section, we conduct a Monte Carlo simulation to evaluate the finite sample performance of the proposed test. As a comparison, we also considere Pesaran and Yamagata's (2008) $\Delta$ test statistics $\hat{\Delta}$ and $\tilde{\Delta}$, and Su and Chen's (2013) residual-based LM test. Pesaran and Yamagata (2008) assume that $u_{i t}$ are mutually uncorrelated over $i$ and $t$. Although Su and Chen (2013) allow cross-sectional dependence through the factor structure among $u_{i t}$, their conditions on the relationship between $N$ and $T$ are stronger than ours.

The $\Delta$ test of Pesaran and Yamagata (2008) is constructed in the following way. Using the individual slope estimator $\hat{\boldsymbol{\beta}}_{i, F E}=\left(X_{i}^{\prime} M_{1} X_{i}\right)^{-1} X_{i}^{\prime} M_{1} \boldsymbol{y}_{i}$, with $M_{1}=I-\mathbf{1 1}^{\prime} / T$ and the weighted pooled estimator $\hat{\boldsymbol{\beta}}_{W F E}=\left(\sum_{i=1}^{N} X_{i}^{\prime} M_{1} X_{i} / \hat{\sigma}_{i}^{2}\right)^{-1} \sum_{i=1}^{N} X_{i}^{\prime} M_{1} \boldsymbol{y}_{i} / \hat{\sigma}_{i}^{2}$ with $\hat{\sigma}_{i}^{2}=\left(\boldsymbol{y}_{i}-X_{i} \hat{\boldsymbol{\beta}}_{i, F E}\right)^{\prime} M_{1}\left(\boldsymbol{y}_{i}-X_{i} \hat{\boldsymbol{\beta}}_{i, F E}\right) /(T-p-1)$, define the $\Delta$ test as

$$
\hat{\Delta}=\sqrt{N}\left(\frac{N^{-1} \hat{S}-p}{\sqrt{2 p}}\right)
$$

where $\hat{S}$ is given as

$$
\hat{S}=\sum_{i=1}^{N}\left(\hat{\boldsymbol{\beta}}_{i, F E}-\hat{\boldsymbol{\beta}}_{W F E}\right)^{\prime}\left(\frac{X_{i}^{\prime} M_{1} X_{i}}{\hat{\sigma}_{i}^{2}}\right)\left(\hat{\boldsymbol{\beta}}_{i, F E}-\hat{\boldsymbol{\beta}}_{W F E}\right) .
$$

Pesaran and Yamagata (2008) also consider a variation to the $\Delta$ test, labeled as $\tilde{\Delta}$. Instead of using $\hat{\sigma}_{i}^{2}$, the $\tilde{\Delta}$ test uses $\tilde{\sigma}_{i}^{2}=\left(\boldsymbol{y}_{i}-X_{i} \hat{\boldsymbol{\beta}}_{F E}\right)^{\prime} M_{1}\left(\boldsymbol{y}_{i}-X_{i} \hat{\boldsymbol{\beta}}_{F E}\right) /(T-1)$. Here, $\hat{\boldsymbol{\beta}}_{F E}=\left(\sum_{i=1}^{N} X_{i}^{\prime} M_{1} X_{i}\right)^{-1} \sum_{i=1}^{N} X_{i}^{\prime} M_{1} \boldsymbol{y}_{i}$. Pesaran and Yamagata (2008) assume the error terms are cross-sectionally and serially independent, and the regressors are strictly exogenous. In the presence of interactive effects (factor errors), the $\Delta$ test works when the regressors are uncorrelated with the factor errors, but will not work when correlations are allowed. 
The LM test of $\mathrm{Su}$ and Chen (2013) is constructed in the following way. First estimate the model under the null of common slope coefficients using the method of Bai (2009) with interactive effects and get the residuals $\hat{\boldsymbol{\varepsilon}}_{i}=\boldsymbol{y}_{i}-X_{i} \hat{\boldsymbol{\beta}}-\hat{F} \hat{\boldsymbol{\lambda}}_{i}$ for $i=1,2, \ldots, N$. Then the LM test is defined as

$$
J_{N T}=\left(L M_{N T}-B_{N T}\right) / V_{N T}^{1 / 2}
$$

where

$$
L M_{N T}=\frac{1}{\sqrt{N}} \sum_{i=1}^{N} \hat{\varepsilon}_{i}^{\prime} X_{i}\left(X_{i}^{\prime} X_{i}\right)^{-1} X_{i}^{\prime} \hat{\varepsilon}_{i} .
$$

and $B_{N T}$ and $V_{N T}$ are estimated by

$$
\hat{B}_{N T}=\frac{1}{\sqrt{N}} \sum_{i=1}^{N} \sum_{t=1}^{T} \hat{\varepsilon}_{i t}^{2} \hat{h}_{i, t t} \quad \text { and } \quad \hat{V}_{N T}=\frac{4}{T^{2} N} \sum_{i=1}^{N} \sum_{t=2}^{T}\left(\hat{\varepsilon}_{i t} \hat{b}_{i t} \sum_{s=1}^{t-1} \hat{b}_{i s} \hat{\varepsilon}_{i s}\right),
$$

where $\hat{h}_{i, t t}$ denotes the $t$-th diagonal element of $\hat{H}_{i}=M_{\hat{F}} X_{i}\left(X_{i}^{\prime} X_{i}\right)^{-1} X_{i}^{\prime} M_{\hat{F}}$, and $\hat{b}_{i t}=$ $\left(X_{i}^{\prime} X_{i} / T\right)^{-1 / 2}\left(\boldsymbol{x}_{i t}-\sum_{s=1}^{T} \hat{\boldsymbol{f}}_{t}^{\prime} \hat{\boldsymbol{f}}_{s} \boldsymbol{x}_{i t}\right)$. The limiting distribution is standard normal under some regularity conditions. The LM test requires that $N^{3 / 4} / T \rightarrow 0$ and $T^{2 / 3} / N \rightarrow 0$. These conditions imply a narrower band between $N$ and $T$ than the conditions in our test, thus the LM test is likely to be sensitive to the sample size.

\subsection{Data generating processes}

GDP1: The first data generating process considered is $y_{i t}=\boldsymbol{x}_{i t}^{\prime} \boldsymbol{\beta}_{i}+u_{i t}$ and $u_{i t}=$ $\boldsymbol{f}_{t}^{\prime} \boldsymbol{\lambda}_{i}+\varepsilon_{i t}$, where the $r(=2)$-dimensional factor $\boldsymbol{f}_{t}$ is a vector of $N(0,1)$ variables, each element of the factor-loading vector $\boldsymbol{\lambda}_{i}$ follows $N(0, I)$, and the noise term $\varepsilon_{i t}$ is also generated from $N(0,1)$. Setting $p=2$, each of the elements of $X_{i}$ is generated from the uniform distribution over $[-2,2]$.

Under the null $H_{0}$, the true parameter vectors $\boldsymbol{\beta}_{i}$ were set to $\boldsymbol{\beta}_{i}=(-1 / 2,1 / 2)^{\prime}$, $i=1, \ldots, N$. Under the alternative $H_{1}$, the true parameter vectors $\boldsymbol{\beta}_{i}$ were set to $\boldsymbol{\beta}_{i}=$ $\left(\beta_{i 1}, 1 / 2\right)^{\prime}$ with $\beta_{i 1}$ being generated from the uniform distribution over $[-0.65,-0.35]$, so the mean is equal to the null value.

GDP2: As the second example, we investigated the performance of the proposed testing procedure when the regressors and the unobservable factor structures have dependency. We generated the regressors as follows:

$$
x_{i t, 1}=0.2+0.3 \boldsymbol{f}_{t}^{\prime} \boldsymbol{\lambda}_{i}+\varepsilon_{i t, 1}^{x}, \quad \text { and } \quad x_{i t, 2}=0.5+0.5 \boldsymbol{f}_{t}^{\prime} \boldsymbol{\lambda}_{i}+\varepsilon_{i t, 2}^{x},
$$

where $\varepsilon_{i t, 1}^{x}$ and $\varepsilon_{i t, 2}^{x}$ are independently generated from the standard normal distribution. The other variables are defined as before. The key feature of this model is that the noise $\boldsymbol{u}_{i}$ and regressors $X_{i}$ are correlated. 


\subsection{Results}

We consider various configurations of $(N, T)$. For a given configuration of $(N, T)$, we generate 1,000 replications from each of the two data generating models. Our test statistics $\hat{\Gamma}$ in (5) and $\tilde{\Gamma}$ in (7) are calculated under the true number of common factors $r=2$ in our testing procedure, as it can be identified by the $C_{p}$ criterion of Ando and Bai (2015).

Theorem 1 suggests that our test statistic $\hat{\Gamma}$ in (5) is asymptotically normal with mean 0 and standard deviation 1, when the null hypothesis of slope homogeneity is satisfied. Therefore, we reject the null hypothesis if the absolute value of our test statistic exceeds the critical values based on the normal distribution. We use the critical value at the nominal level of $\alpha=5 \%$ (1.96). We demonstrate that our proposed test has the correct size and satisfactory power. Furthermore, we check the finite sample power rejection frequency of our testing procedure under the alternative.

The finite sample properties of the proposed test under each of the data generating processes are summarized in Tables 1-3. Each column reports the rejection frequency under the null $H_{0}$ and the alternative $H_{1}$. Table 1 provides the size and power under the first data generating process with a wide range of $N$ and $T$. The results for $\hat{\Delta}$ and $\tilde{\Delta}$ are in line with those of Pesaran and Yamagata (2008). Furthermore, the LM results are in line with those of $\mathrm{Su}$ and Chen (2013).

Table 1 suggests that the level of our test behaves reasonably well as $N$ and $T$ increase. When the null hypothesis does not hold, Table 1 suggests our test statistics $\hat{\Gamma}$ and $\tilde{\Gamma}$ have higher power than those of $\hat{\Delta}$ and $\tilde{\Delta}$ under small $T$ and $N$.

However, we can see the clear advantages of our testing procedure in Table 2. Under the null, Pesaran and Yamagata's (2008) testing procedure rejects the null in all cases. In contrast, our testing procedure has nice size control and power for all combinations of $N$ and $T$. This difference arises because $\boldsymbol{x}_{i t}$ and the factor structure are correlated in the second data generating process. Our test permits this correlation.

$\mathrm{Su}$ and Chen's (2013) procedure works well in general. There is, however, a tendency of size distortion when $N$ is relatively large with respect to $T$, for example, $T=50$ and $N=200$. A possible reason is that their test imposes the following conditions: $N^{3 / 4} / T \rightarrow 0$ and $T^{2 / 3} / N \rightarrow 0$ as $N, T \rightarrow \infty$. In general, these conditions are sufficient, and they are not necessary. Nevertheless, they imply a relatively narrow band between $N$ and $T$. To verify this, we further compared the size and power of all tests under large N. Under much larger $N$ relative to $T$, the finite sample properties of the proposed test are summarized in Table 3. Under the second data generating process with $(N, T)=$ $(200,20)$, the finite sample rejection frequency at the $\alpha=5 \%$ nominal level for our test is 6.9 under the null $H_{0}$ and 24.0 under the alternative $H_{1}$. However, those of $\mathrm{Su}$ and Chen's (2013) test are 30.2 under the null $H_{0}$ and 73.3 under the alternative $H_{1}$. It can be seen that our proposed test still provides correct size, while a size 
distortion is observed with $\mathrm{Su}$ and Chen's (2013) test. This difference arises because of the relationship between $N$ and $T$. Similar observations can be made for Pesaran and Yamagata's (2008) $\hat{\Delta}$ test statistic. As $\tilde{\Delta}$ needs the weaker condition $\sqrt{N} / T \rightarrow 0$, the $\tilde{\Delta}$ procedure has nice size control under the first DGP, but not under the second DGP, as before. Our test continues to work well under much larger $N$ than $T$.

\section{Conclusion}

In this paper we examined the problem of testing slope homogeneity in a high-dimensional panel data model. We developed testing procedures based on the Swamy's (1970) test principle. Our testing procedure allows cross-sectional dependence as well as the dependence between the regressors and unobservable factor structures. Monte Carlo experiments suggest that the proposed method is a useful testing procedure for detecting slope homogeneity in high-dimensional panel data in which both the time dimension and the cross-sectional dimension are large.

\section{References}

[1] Ando, T. \& Bai, J. (2015) Asset pricing with a general multifactor structure. Journal of Financial Econometrics, 13, 556-604.

[2] Bai, J. (2009) Panel data models with interactive fixed effects. Econometrica 77, $1229-1279$.

[3] Bai, J. \& Ng, S. (2002) Determining the number of factors in approximate factor models. Econometrica 70, 191-221.

[4] Blomquist, J. \& Westerlund, J. (2013) Testing slope homogeneity in large panels with serial correlation. Economics Letters 121, 374-378

[5] Carhart, M. M. (1997) On persistence in mutual fund performance. Journal of Finance 52, 57-82.

[6] Connor, G. \& Korajczyk, R. (1986) Performance measurement with the arbitrage pricing theory: a new framework for analysis. Journal of Financial Economics 15, $3730-3794$.

[7] Fama, E. \& French, K. (1992) The cross-section of expected stock returns. Journal of Finance 47, 427-465.

[8] Lintner, J. (1965) The Valuation of Risk Assets and the Selection of Risky Investments in Stock Portfolios and Capital Budgets. Review of Economics and Statistics, 47:1, 13-37 
[9] Pesaran, M. H. \& Yamagata, T. (2008) Testing slope homogeneity in large panels. Journal of Econometrics 142, 50-93.

[10] Pesaran, H., Smith, R. \& Im, K. S. (1996) Dynamic linear models for heterogenous panels. In: Matyas, L. \& Sevestre, P. (Eds.), Econometrics of Panel Data: A Handbook of the Theory with Applications, second revised edition. Kluwer Academic Publishers, Dordrecht, 145-195.

[11] Phillips, P. C. B. \& Sul, D. (2003) Dynamic panel estimation and homogeneity testing under cross section dependence. Econometrics Journal 6, 217-259.

[12] Sharpe, W.F. (1964) Capital Asset Prices: A Theory of Market Equilibrium under Conditions of Risk. Journal of Finance. 19:3, 425-442.

[13] Song, M. (2013) Essays on Large Panel Data Analysis. Ph.D. Thesis, Columbia University.

[14] Su, L. \& Chen, Q. (2013) Testing homogeneity in panel data models with interactive fixed effects. Econometric Theory 29, 1079-1135

[15] Swamy, P. A. V. B. (1970). Efficient inference in a random coefficient regression model. Econometrica 38, 311-323.

Appendix 1: Proof of Theorem 1. Let $\hat{\boldsymbol{\beta}}_{i}$ be obtained by minimizing the leastsquares objective function in (4). Let $S_{i i}=\left(X_{i}^{\prime} M_{F^{0}} X_{i}\right) / T, L_{i j}=a_{i j}\left(X_{i}^{\prime} M_{F^{0}} X_{j}\right) / T$ with $a_{i j}=\boldsymbol{\lambda}_{j}^{0^{\prime}}\left(\Lambda^{0^{\prime}} \Lambda^{0} / N\right)^{-1} \boldsymbol{\lambda}_{j}^{0}$ and $\boldsymbol{\zeta}_{i}=X_{i}^{\prime} M_{F^{0}} \varepsilon_{i} / \sqrt{T}$. As in Song (2013), it can be shown that under $\sqrt{T} / N \rightarrow 0$,

$$
\sqrt{T}\left(\hat{\boldsymbol{\beta}}_{i}-\boldsymbol{\beta}_{i}^{0}\right)=S_{i i}^{-1} \boldsymbol{\zeta}_{i}+S_{i i}^{-1} \frac{1}{N} \sum_{j=1}^{N} L_{i j} \sqrt{T}\left(\hat{\boldsymbol{\beta}}_{j}-\boldsymbol{\beta}_{j}^{0}\right)+o_{p}(1), \quad i=1, \ldots, N,
$$

From (10), stacking the estimators across $i$,

$$
\begin{aligned}
& =\left(\begin{array}{c}
\hat{\boldsymbol{\beta}}_{1}-\boldsymbol{\beta}_{1}^{0} \\
\hat{\boldsymbol{\beta}}_{2}-\boldsymbol{\beta}_{2}^{0} \\
\vdots \\
\hat{\boldsymbol{\beta}}_{N}-\boldsymbol{\beta}_{N}^{0}
\end{array}\right)=\left(\begin{array}{cccc}
S_{11}^{-1} & & & \\
& S_{22}^{-1} & & \\
& & \ddots & \\
& & S_{N N}^{-1}
\end{array}\right)\left(\begin{array}{c}
\boldsymbol{\zeta}_{1} \\
\boldsymbol{\zeta}_{2} \\
\vdots \\
\boldsymbol{\zeta}_{N}
\end{array}\right) \\
+ & +\frac{1}{N}\left(\begin{array}{ccccc}
S_{11}^{-1} & & \\
& S_{22}^{-1} & & \\
& & \ddots & \\
& & S_{N N}^{-1}
\end{array}\right)\left(\begin{array}{cccc}
L_{11} & L_{12} & \cdots & L_{1 N} \\
L_{21} & L_{22} & \ddots & L_{2 N} \\
\vdots & \ddots & \ddots & \vdots \\
L_{N 1} & L_{N 2} & \cdots & L_{N N}
\end{array}\right)\left(\begin{array}{c}
\sqrt{T}\left(\hat{\boldsymbol{\beta}}_{1}-\boldsymbol{\beta}_{1}^{0}\right) \\
\sqrt{T}\left(\hat{\boldsymbol{\beta}}_{2}-\boldsymbol{\beta}_{2}^{0}\right) \\
\vdots \\
\sqrt{T}\left(\hat{\boldsymbol{\beta}}_{N}-\boldsymbol{\beta}_{N}^{0}\right)
\end{array}\right)+o_{p}(1) .
\end{aligned}
$$


We can express the above formula as

$$
\sqrt{T}\left(\hat{\boldsymbol{\beta}}-\boldsymbol{\beta}^{0}\right)=S^{-1} \boldsymbol{\zeta}+\frac{1}{N} S^{-1} L \sqrt{T}\left(\hat{\boldsymbol{\beta}}-\boldsymbol{\beta}^{0}\right)+o_{p}(1),
$$

where $\hat{\boldsymbol{\beta}}^{\prime}=\left(\hat{\boldsymbol{\beta}}_{1}^{\prime}, \ldots, \hat{\boldsymbol{\beta}}_{N}^{\prime}\right), \boldsymbol{\beta}^{0^{\prime}}=\left(\boldsymbol{\beta}_{1}^{0^{\prime}}, \ldots, \boldsymbol{\beta}_{N}^{0 \prime}\right), \boldsymbol{\zeta}^{\prime}=\left(\boldsymbol{\zeta}_{1}^{\prime}, \ldots, \boldsymbol{\zeta}_{N}^{\prime}\right), S$ is an $N p \times N p$ block diagonal matrix with $i$ th block $S_{i i}$, and $L$ is an $N p \times N p$ matrix with $i j$-th block $L_{i j}$. The above can be further written as

$$
\left(S-\frac{1}{N} L\right) \sqrt{T}\left(\hat{\boldsymbol{\beta}}-\boldsymbol{\beta}^{0}\right)=\boldsymbol{\zeta}+o_{p}(1),
$$

Let $\Omega$ be the variance-covariance matrix of $\boldsymbol{\zeta}$, as defined in Assumption F. From (12), we then have

$$
\Omega^{-1 / 2}\left(S-\frac{1}{N} L\right) \sqrt{T}\left(\hat{\boldsymbol{\beta}}-\boldsymbol{\beta}^{0}\right)=\Omega^{-1 / 2} \boldsymbol{\zeta}+o_{p}(1)
$$

Taking its quadratic form, subtracting $N p$ on each side and dividing by $\sqrt{2 N p}$, we get

$$
\frac{T\left(\hat{\boldsymbol{\beta}}-\boldsymbol{\beta}^{0}\right)^{\prime}\left(S-\frac{1}{N} L^{\prime}\right) \Omega^{-1}\left(S-\frac{1}{N} L\right)\left(\hat{\boldsymbol{\beta}}-\boldsymbol{\beta}^{0}\right)-N p}{\sqrt{2 N p}}=\frac{\boldsymbol{\zeta}^{\prime} \Omega^{-1} \boldsymbol{\zeta}-N p}{\sqrt{2 N p}}+o_{p}(1) .
$$

By Assumption F,

$$
\frac{\boldsymbol{\zeta}^{\prime} \Omega^{-1} \boldsymbol{\zeta}-N p}{\sqrt{2 N p}} \rightarrow N(0,1) .
$$

So the left hand side of $(13)$ is asymptotically $N(0,1)$. It can be shown that replacing $\boldsymbol{\beta}^{0}$ by $\overline{\boldsymbol{\beta}}_{1_{N}}$, and the unknown elements in $S, L$, and $\Omega$ by their estimators, the same asymptotic representation holds. This gives the result of Theorem 1.

Appendix 2: Proof of (7). The test statistic $\hat{\Gamma}$ is derived by correcting bias for the whole system (joint bias). If we correct the bias for each individual parameter $\hat{\boldsymbol{\beta}}_{i}$, then using

$$
\frac{1}{N} S_{i i}^{-1} L_{i i}=\frac{1}{N} a_{i i} I_{p}=\boldsymbol{\lambda}_{i}^{0^{\prime}}\left(\Lambda^{0^{\prime}} \Lambda^{0}\right)^{-1} \boldsymbol{\lambda}_{i}^{0} I_{p}
$$

we have

$$
\sqrt{T}\left(\hat{\boldsymbol{\beta}}_{i}-\boldsymbol{\beta}_{i}^{0}\right)=S_{i i}^{-1} \boldsymbol{\zeta}_{i}+\frac{1}{N} a_{i i} \sqrt{T}\left(\hat{\boldsymbol{\beta}}_{i}-\boldsymbol{\beta}_{i}^{0}\right)+o_{p}(1) .
$$

The reason that this approximation works is that the weighted average of $\left(\hat{\boldsymbol{\beta}}_{j}-\boldsymbol{\beta}_{j}^{0}\right)$ for $j \neq i$,

$$
S_{i i}^{-1} \frac{1}{N} \sum_{j \neq i} L_{i j} \sqrt{T}\left(\hat{\boldsymbol{\beta}}_{j}-\boldsymbol{\beta}_{j}^{0}\right),
$$

is of small magnitude, and is also uncorrelated with the leading term $\zeta_{i}$. This leads to

$$
S_{i i} \sqrt{T}\left(\hat{\boldsymbol{\beta}}_{i}-\boldsymbol{\beta}_{i}^{0}\right)\left(1-a_{i i} / N\right)=\boldsymbol{\zeta}_{i}+o_{p}(1),
$$


or

$$
\Omega_{i}^{-1 / 2} S_{i i} \sqrt{T}\left(\hat{\boldsymbol{\beta}}_{i}-\boldsymbol{\beta}_{i}^{0}\right)\left(1-a_{i i} / N\right)=\Omega_{i}^{-1 / 2} \boldsymbol{\zeta}_{i}+o_{p}(1)
$$

We then have

$$
T\left(\hat{\boldsymbol{\beta}}_{i}-\boldsymbol{\beta}_{i}^{0}\right)^{\prime} S_{i i} \Omega^{-1} S_{i i}\left(\hat{\boldsymbol{\beta}}_{i}-\boldsymbol{\beta}_{i}^{0}\right)\left(1-a_{i i} / N\right)^{2}=\boldsymbol{\zeta}_{i}^{\prime} \Omega_{i}^{-1} \boldsymbol{\zeta}_{i}+o_{p}(1),
$$

for $i=1, \ldots, N$. The rest argument is similar to the previous case, and the asymptotic normality of the test follows from Assumption F. This gives (7). 
Table 1: Finite sample properties of the proposed test under the first data generating process. Each column reports the rejection frequency (\%) under the null $H_{0}$ and the alternative $H_{1}$. Critical level is set at $\alpha=5 \% . \hat{\Gamma}$ : the proposed test statistic in (5), $\tilde{\Gamma}$ : the proposed test statistic in (7), $\hat{\Delta}$ : the test procedure of Pesaran and Yamagata (2008), $\tilde{\Delta}$ : the test procedure of Pesaran and Yamagata (2008) and LM: the residualbased Lagrangian Multiplier test of Su and Chen (2013).

\begin{tabular}{rrrrrrr}
\multicolumn{8}{c}{ Rejection frequency $(\%)$ under $H_{0}$} \\
\hline$T$ & $N$ & $\hat{\Gamma}$ & $\tilde{\Gamma}$ & $\tilde{\Delta}$ & $\hat{\Delta}$ & LM \\
\hline 50 & 50 & 5.0 & 5.1 & 4.2 & 6.9 & 5.8 \\
50 & 100 & 5.6 & 5.4 & 3.5 & 7.5 & 6.6 \\
50 & 200 & 5.2 & 5.3 & 3.8 & 11.0 & 7.8 \\
100 & 50 & 6.0 & 6.0 & 3.9 & 4.8 & 4.9 \\
100 & 100 & 5.7 & 5.8 & 4.8 & 6.4 & 5.6 \\
100 & 200 & 5.2 & 5.2 & 4.1 & 6.2 & 5.4 \\
200 & 50 & 5.6 & 5.6 & 4.7 & 5.3 & 5.0 \\
200 & 100 & 5.2 & 5.2 & 4.6 & 5.1 & 5.3 \\
200 & 200 & 5.0 & 5.0 & 4.5 & 4.9 & 5.4 \\
\hline
\end{tabular}

\begin{tabular}{rrrrrrr}
\multicolumn{8}{c}{ Rejection frequency $(\%)$ under $H_{1}$} \\
\hline$T$ & $N$ & $\hat{\Gamma}$ & $\tilde{\Gamma}$ & $\tilde{\Delta}$ & $\hat{\Delta}$ & LM \\
\hline 50 & 50 & 23.0 & 23.8 & 9.5 & 20.4 & 39.6 \\
50 & 100 & 50.1 & 49.9 & 14.7 & 32.2 & 70.0 \\
50 & 200 & 83.1 & 83.2 & 30.3 & 60.3 & 95.7 \\
100 & 50 & 70.8 & 71.3 & 31.1 & 37.8 & 83.1 \\
100 & 100 & 95.4 & 95.5 & 52.8 & 62.0 & 97.3 \\
100 & 200 & 100.0 & 100.0 & 80.8 & 89.1 & 100.0 \\
200 & 50 & 98.7 & 98.7 & 72.2 & 76.3 & 99.4 \\
200 & 100 & 100.0 & 100.0 & 94.4 & 95.7 & 100.0 \\
200 & 200 & 100.0 & 100.0 & 100.0 & 100.0 & 100.0 \\
\hline
\end{tabular}


Table 2: Finite sample properties of the proposed test under the second data generating process. Each column reports the rejection frequency (\%) under the null $H_{0}$ and the alternative $H_{1}$. Critical level is set at $\alpha=5 \% . \hat{\Gamma}$ : the proposed test statistic in (5), $\tilde{\Gamma}$ : the proposed test statistic in (7), $\hat{\Delta}$ : the test procedure of Pesaran and Yamagata (2008), $\tilde{\Delta}$ : the test procedure of Pesaran and Yamagata (2008) and LM: the residualbased Lagrangian Multiplier test of Su and Chen (2013).

\begin{tabular}{rrrrrrr}
\multicolumn{8}{c}{ Rejection frequency $(\%)$ under $H_{0}$} \\
\hline \multicolumn{1}{c}{$N$} & $N$ & $\hat{\Gamma}$ & $\tilde{\Gamma}$ & $\tilde{\Delta}$ & $\hat{\Delta}$ & LM \\
\hline 50 & 50 & 5.2 & 4.9 & 100.0 & 100.0 & 5.0 \\
50 & 100 & 5.8 & 5.9 & 100.0 & 100.0 & 7.8 \\
50 & 200 & 4.9 & 5.0 & 100.0 & 100.0 & 7.6 \\
100 & 50 & 5.4 & 5.4 & 100.0 & 100.0 & 5.1 \\
100 & 100 & 5.0 & 5.0 & 100.0 & 100.0 & 5.3 \\
100 & 200 & 4.6 & 4.5 & 100.0 & 100.0 & 5.4 \\
200 & 50 & 5.2 & 5.2 & 100.0 & 100.0 & 4.8 \\
200 & 100 & 4.6 & 4.4 & 100.0 & 100.0 & 4.5 \\
200 & 200 & 5.3 & 5.5 & 100.0 & 100.0 & 5.2 \\
\hline
\end{tabular}

\begin{tabular}{rrrrrrr}
\multicolumn{8}{c}{ Rejection frequency $(\%)$} & under $H_{1}$ \\
\hline$T$ & $N$ & $\hat{\Gamma}$ & $\tilde{\Gamma}$ & $\tilde{\Delta}$ & $\hat{\Delta}$ & LM \\
\hline 50 & 50 & 18.1 & 18.8 & 100.0 & 100.0 & 33.1 \\
50 & 100 & 37.1 & 37.8 & 100.0 & 100.0 & 54.7 \\
50 & 200 & 61.2 & 61.6 & 100.0 & 100.0 & 82.2 \\
100 & 50 & 51.5 & 51.5 & 100.0 & 100.0 & 65.8 \\
100 & 100 & 88.5 & 88.6 & 100.0 & 100.0 & 94.0 \\
100 & 200 & 98.8 & 98.6 & 100.0 & 100.0 & 99.9 \\
200 & 50 & 96.2 & 96.2 & 100.0 & 100.0 & 97.5 \\
200 & 100 & 99.8 & 99.8 & 100.0 & 100.0 & 100.0 \\
200 & 200 & 100.0 & 100.0 & 100.0 & 100.0 & 100.0 \\
\hline
\end{tabular}


Table 3: Finite sample properties of the proposed test when $N$ is large relative to $T$. Each column reports the rejection frequency (\%) under the null $H_{0}$ and the alternative $H_{1}$. Critical level is set at $\alpha=5 \% . \hat{\Gamma}$ : the proposed test statistic in $(5), \tilde{\Gamma}$ : the proposed test statistic in (7), $\hat{\Delta}$ : the test procedure of Pesaran and Yamagata (2008), $\tilde{\Delta}$ : the test procedure of Pesaran and Yamagata (2008) and LM: the residual-based Lagrangian Multiplier test of Su and Chen (2013).

\begin{tabular}{lcrrrrrr}
\multicolumn{1}{l}{ Rejection frequency under $H_{0}$} \\
\hline & $T$ & $N$ & $\hat{\Gamma}$ & $\tilde{\Gamma}$ & $\tilde{\Delta}$ & $\hat{\Delta}$ & LM \\
\hline DGP 1 & 20 & 200 & 6.9 & 6.9 & 3.1 & 43.9 & 30.2 \\
& 50 & 500 & 6.3 & 6.1 & 4.3 & 17.9 & 14.8 \\
& 50 & 800 & 5.2 & 5.0 & 3.7 & 26.2 & 20.4 \\
DGP 2 & 20 & 200 & 6.9 & 7.0 & 100.0 & 100.0 & 30.8 \\
& 50 & 500 & 5.5 & 5.6 & 100.0 & 100.0 & 15.7 \\
& 50 & 800 & 4.9 & 4.9 & 100.0 & 100.0 & 21.4 \\
\hline
\end{tabular}

Rejection frequency under $H_{1}$

\begin{tabular}{rrrrrrrr}
\hline & $T$ & $N$ & $\hat{\Gamma}$ & $\tilde{\Gamma}$ & $\tilde{\Delta}$ & $\hat{\Delta}$ & $\mathrm{LM}$ \\
\hline DGP 1 & 20 & 200 & 24.0 & 24.4 & 5.3 & 66.2 & 73.4 \\
& 50 & 500 & 99.7 & 99.7 & 57.5 & 90.1 & 100.0 \\
& 50 & 800 & 100.0 & 100.0 & 81.9 & 98.2 & 100.0 \\
DGP 2 & 20 & 200 & 16.8 & 17.3 & 100.0 & 100.0 & 67.1 \\
& 50 & 500 & 96.3 & 96.2 & 100.0 & 100.0 & 99.6 \\
& 50 & 800 & 99.8 & 99.8 & 100.0 & 100.0 & 100.0 \\
\hline
\end{tabular}

